\title{
Chronische Beschwerden des Bewegungsorgans Bedeutung der Osteoporose bei rheumatischen Erkrankungen
}

\author{
H. W. Minne, M. Pfeifer, W. Pollähne, Institut für Klinische Osteologie „Gustav Pommer“ und Klinik „Der Fürstenhof“, Bad Pyrmont
}

NOTFALLMEDIZIN 2003, 29: 347-355

Derzeit leben in Deutschland etwa zwei Millionen Frauen mit bereits eingetretenen Wirbelkörpereinbrüchen sowie mehr als 800000 Männer mit derartigen Frakturen. 40\% der weiblichen Patienten mit rheumatoider Arthritis weisen eine Osteopenie, 20\% eine Osteoporose auf. 80\% aller Patienten mit rheumatoider Arthritis sind älter als 50 Jahre. Wirbelfrakturen sind bei einem zurzeit noch nicht abschätzbaren Anteil der Patienten mit den schlimmst vorstellbaren Schmerzen assoziiert und führen zu einschneidenden Einschränkungen der Leistungsfähigkeit im Rahmen der häuslichen Selbstversorgung. Die Knochendichteanalyse ist nur ein einzelner Mosaikstein bei der Abklärung einer Osteoporose, sie ist jedoch nach wie vor das einzige Verfahren, mit dessen Hilfe das Risiko zukünftiger Frakturen bestimmt werden kann. Als Behandlungsoptionen stehen sowohl Medikamente zur Verfügung als auch nichtpharmakologische Maßnahmen, welche in den Leitlinien des Dachverbandes deutschsprachiger osteologischer Fachgesellschaften (DVO) enthalten sind.

$\mathrm{R}$ heumatismus: Gruppenbegriff (ohne diagnostische Wertigkeit) für „schmerzhafte und funktionsbeeinträchtigende $\mathrm{Zu}$ stände des Muskel-Skelettsystems unter Einschluss der sie begleitenden oder auch isoliert auftretenden Vorgänge an anderen Organsystemen" (Handlexikon der Medizin, Urban und Schwarzenberg, München - Wien - Baltimore).

Der Begriff der „rheumatischen Erkrankungen“ deckt also nicht allein die klassisch entzündlichen Krankheiten der Gelenke, sondern jedwede Krankheitsformen, die mit chronischen Beschwerden der verschiedenen Gewebe des Bewegungsorgans verbunden sind.

\section{Kasuistik anstelle einer \\ Einleitung}

Ein 52-jähriger Mann leidet unter zunehmenden chronischen Schmerzen, die zunächst in den Bereich der Wirbelsäule und der rechten Schulter projiziert werden und im weiteren Verlauf auch mehrere große und kleine Gelenke des Körpers befallen. Zunächst erfolgt eine topische Applikation von Glu- kokortikoiden, die später auch als systemische Behandlung oral und parenteral verabreicht werden.

Nach sechs Jahren, also im Alter von 58 Jahren, stürzt der Patient aus zwei Metern Höhe von einer Leiter. Anschließend wird radiologisch die Diagnose von drei Wirbelbrüchen gestellt. Ein halbes Jahr später kommt es im Rahmen einer Schreckreaktion nach Stolpern zu akut neu auftretenden Rückenschmerzen. Radiologisch wird die Diagnose einer weiteren Fraktur gestellt. Im Abschlussbericht nach unfallchirurgischer Betreuung findet sich kein Hinweis auf die Möglichkeit, dass eine Osteoporose zur Teilursache des Bruches geworden war.

Während des nachfolgenden Rehabilitationsverfahrens wird erstmals die Diagnose einer Osteoporose genannt. Es wird eine Behandlung mit dem Bisphosphonat Fosamax $^{\circledR}$ begonnen. Zum Zeitpunkt des Behandlungsbeginnes handelte es sich hierbei um eine so genannte „off-label-Indikation“, weil Fosamax ${ }^{\circledR}$ zum damaligen Zeitpunkt für die Therapie der Osteoporose des Mannes noch nicht zugelassen war und

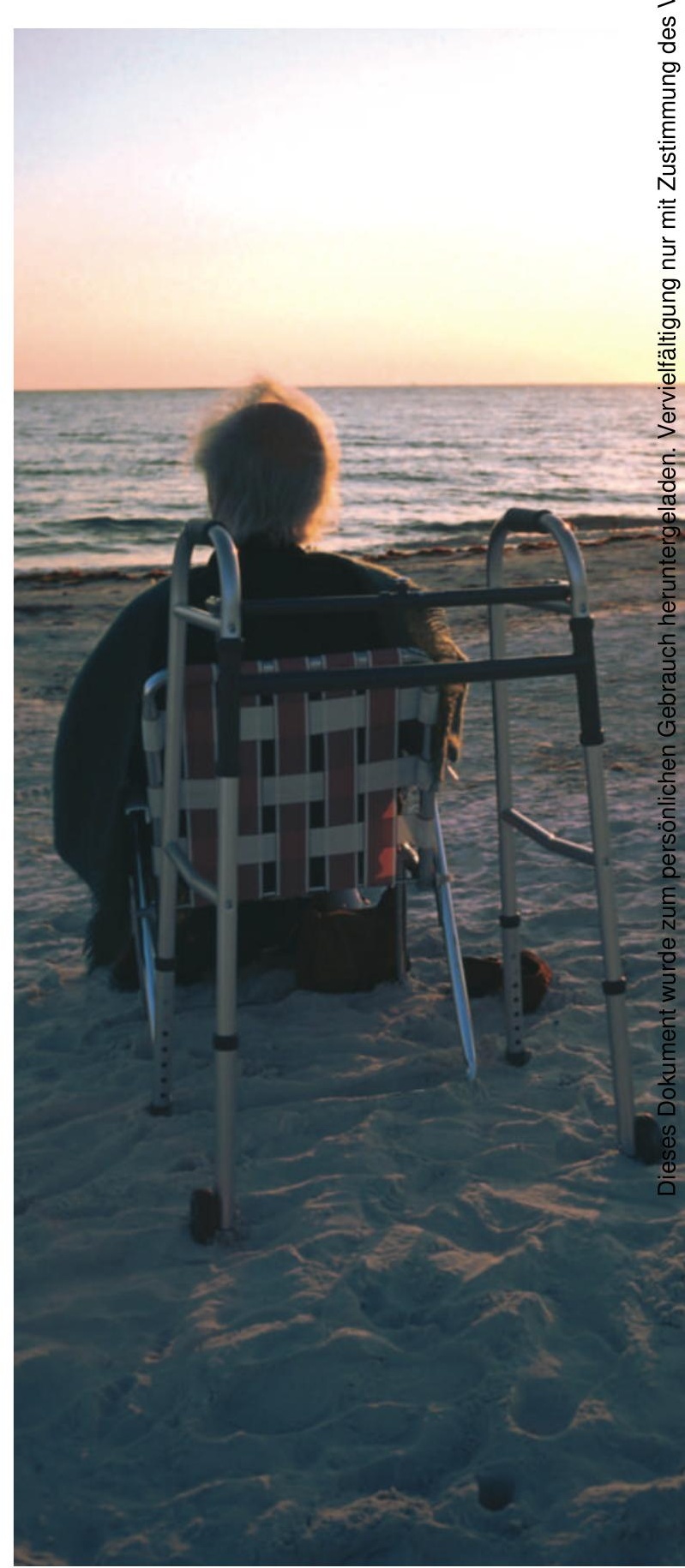


auch keine Zulassung für die Therapie einer steroidabhängig entstandenen Osteoporose hatte. Seit Behandlungsbeginn sind neue Frakturen nicht mehr aufgetreten. Die jedoch bereits stattgefundenen Frakturen beeinträchtigten die Funktion des Achsenskelettes in einem derartigen Ausmaß, dass der Patient in seinem Beruf als Straßenbauer langzeitig arbeitsunfähig wurde und nach zwei weiteren Jahren erwerbsunfähig in Rente ging.

\section{Kassen bremsen Umsetzung neuer Leitlinien}

Inzwischen hat der Dachverband deutschsprachiger osteologischer Fachgesellschaften Leitlinien zur Diagnostik und Therapie der kortikoid-induzierten Osteoporose entwickelt. Diese Leitlinien wurden Anfang des Jahres 2003 durch die Mitgliedsgesellschaften des Dachverbandes aller in Deutschland relevanten Fachgesellschaften, die sich mit Krankheiten des Skelettsystems beschäftigen, verabschiedet.

Die Umsetzung eines Teiles dieser Leitlinien, nämlich die Früherfassung eines Osteoporoserisikos unter Glukokortikoidtherapie mittels Knochendichteanalytik unter Einsatz eines DEXA-Gerätes (DualEnergy-X-ray-Absorptiometry), ist immer noch von Hindernissen umlagert, unter anderem weil die Bestimmung des Knochenmineralgehaltes vor Auftritt eines ersten Knochenbruches als Kassenleistung durch Beschluss der Bundeskommission - der Gesetzeskraft erhalten hat - nach wie vor unzulässig ist.

Es darf also nicht erstaunen, dass in Deutschland nach wie vor weiterhin viele der Patienten, die im Zusammenhang mit der Behandlung einer rheumatischen Krankheit Glukokortikoide erhalten, im Hinblick auf die Früherfassung eines Osteoporose-Risikos und auf eine adäquate anti-osteoporotische Therapie bei Nachweis einer steroidabhängig entstehenden Osteoporose unterversorgt sind. Es rächt sich jetzt, dass in der Vergangenheit die im Zusammenhang mit einer Osteoporose auftretenden sozialmedizinischen und gesundheitsökonomischen Folgen ignoriert wurden.
Epidemiologie der

Osteoporose

Während der vergangenen Jahre wurden umfangreiche epidemiologische Untersuchungen in Europa und den USA durchgeführt, deren Ergebnisse die sozialmedizinische Bedeutung der Krankheit eindrucksvoll belegen.

Den Ergebnissen der europaweit durchgeführten EPOS${ }^{1}$-Studie zufolge leben in Deutschland etwa zwei Millionen Frauen mit bereits eingetretenen Wirbelkörpereinbrüchen sowie mehr als 800000 Männer mit derartigen Frakturen, die als klassische Spätkomplikation einer Osteoporose anzusprechen sind (1). Die Ergebnisse dieser Studie belegen, dass in Deutschland jährlich mehr als 200000 Männer und Frauen von neuen Wirbelkörpereinbrüchen geplagt werden.

Eine eigene Untersuchung, bei der eine Stichprobe von $2 \%$ der deutschen Bevölkerung mit Unterstützung von Betriebskrankenkassen untersucht wurde, zeigte, dass jährlich 130000 Frauen und Männer frische Oberschenkelhalsbrüche erleben (2). Die Zahl der distalen Radiusfrakturen wird mit $60000-$ 80000 pro Jahr geschätzt. Präzise Zahlen zu der Häufigkeit der übrigen, ebenfalls im Zusammenhang mit Osteoporosen auftretenden Brüche (proximale Humerusfraktur, Sprunggelenksfrakturen, Rippenbrüche, u.a.m.) stehen nicht zur Verfügung.

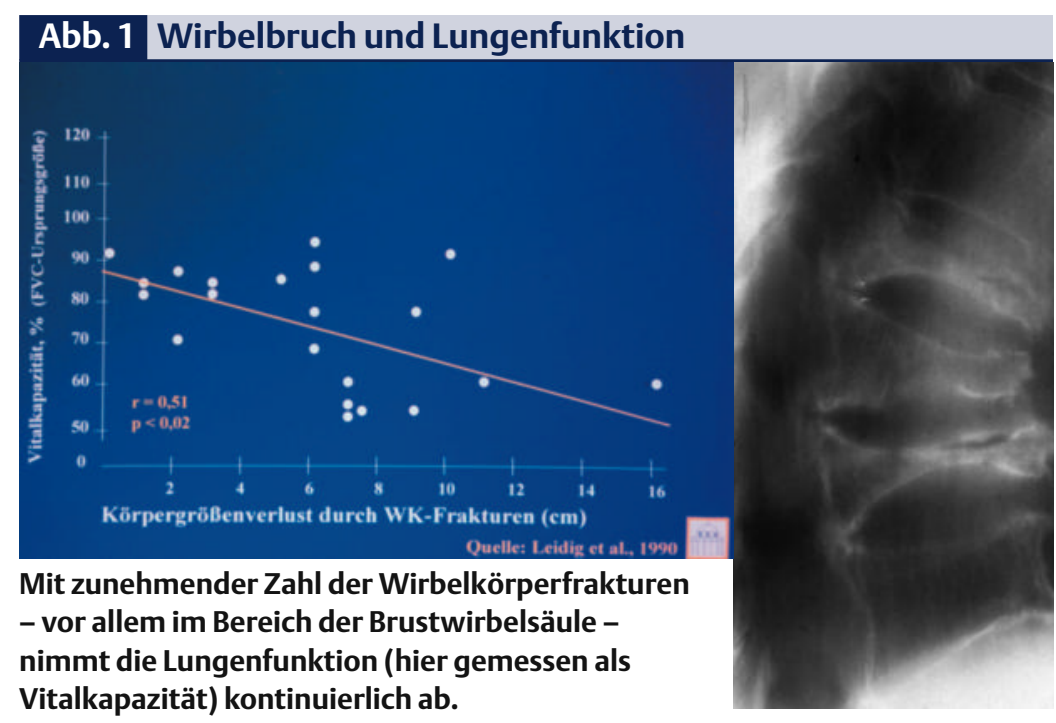

Die Ergebnisse der in Deutschland und im europäischen Ausland durchgeführten Untersuchungen zeigen, dass 20-25\% der an den Folgen einer Osteoporose leidenden Patienten männlichen Geschlechtes sind. Schon vor Jahren haben die Ergebnisse japanischer Untersuchungen gezeigt, dass mit zunehmender durchschnittlicher Lebenserwartung die Inzidenz osteoporotischer Frakturen, besonders deutlich erkennbar beim männlichen Geschlecht, weiterhin zunehmen wird.

Ursprünglich wurde angenommen, dass die Zahl der Oberschenkelhalsbrüche in Europa sich bis zum Jahr 2050 verdoppeln würde. Eine Erhebung der „International Osteoporosis Foundation“ (IOF) zeigte jedoch, dass bereits während eines 3-jährigen Erhebungszeitraumes bis zum Jahr 2002 die Zahl der Oberschenkelhalsbrüche um 25\% zugenommen hatte. Man wird also den Zeitpunkt, zu dem sich die Zahl der Oberschenkelhalsbrüche verdoppelt haben wird, um Jahrzehnte vorverlegen müssen.

Bezogen auf den Zusammenhang zwischen Osteoporose und rheumatischen Erkrankungen sind folgende Daten wichtig: $80 \%$ aller Patienten mit rheumatoider Arthritis (RA) sind älter als 50 Jahre und 40\% der weiblichen Patienten mit RA weisen eine Osteopenie, 20\% eine Osteoporose auf. 15 bis 20\% aller Patienten mit RA erhalten eine Glukokortikoidtherapie (siehe Leitlinien der DVO unter www.bergmannsheil/leitlinien-dvo). 
Sozialmedizinische Folgen

Während der ersten einer klinischen Wirbelfraktur folgenden sechs Monate (klinische Fraktur: Wirbelbruch, der wegen der akuten Beschwerden zum Arztbesuch führt) steigt das Risiko vorzeitigen Sterbens im Vergleich zu Unbetroffenen um mehr als das Achtfache an. Bei Patienten mit Oberschenkelhalsbrüchen findet sich ein ähnlicher Anstieg des Risikos vorzeitigen Sterbens. Bei OberschenkelhalsbruchPatienten wird während der ersten zwölf dem Ereignis folgenden Monate eine Exzessmortalität von mehr als $20 \%$ beobachtet (3). Häufig tritt der Tod als Folge der Akzentuierung der Multimorbidität durch frakturbedingte Schmerzen, Bettlägerigkeit, Pflegebedürftigkeit ein. So ließ sich unter anderem zeigen, dass Patienten mit Wirbelkörpereinbrüchen Einbußen bei der Vitalkapazität und der Sekundenkapazität hinzunehmen haben (Abb. 1) (4). Die Größe der Dunkelziffer bei der Bewertung osteoporosebedingter Mortalität wird anhand der Ergebnisse einer jüngst veröffentlichten skandinavischen Studie deutlich: So war das Risiko des Sterbens an scheinbar ausschließlich kardiovaskulären Krankheiten bei Patienten mit zwei und mehr Wirbelkörpereinbrüchen ein vielfaches höher als das Risiko vorzeitigen Sterbens an scheinbar kardiovaskulären Ursachen bei Patienten ohne Wirbelkörpereinbruch (5).

Wirbelfrakturen sind bei einem zurzeit noch nicht abschätzbaren Anteil der Patienten mit den schlimmst vorstellbaren Schmerzen assoziiert und führen dann zur Pflegebedürftigkeit, die sich über Wochen hinziehen kann. Auch hier findet sich eine sehr hohe Dunkelziffer, da viele Patienten beim zweiten oder dritten Wirbelbruch medizinische Hilfe nicht mehr suchen, weil sie bei der ersten Fraktur die Erfahrung machen mussten, dass ihnen ihre Beschwerden nicht geglaubt wurden, dass ihnen Sozialschmarotzertum oder aber eine pathologische Schmerzwahrnehmung eher psychosomatischen Ursprunges unterstellt wurde (6).

Nach wie vor gilt, dass bei der Mehrzahl derartiger Frakturpatien-

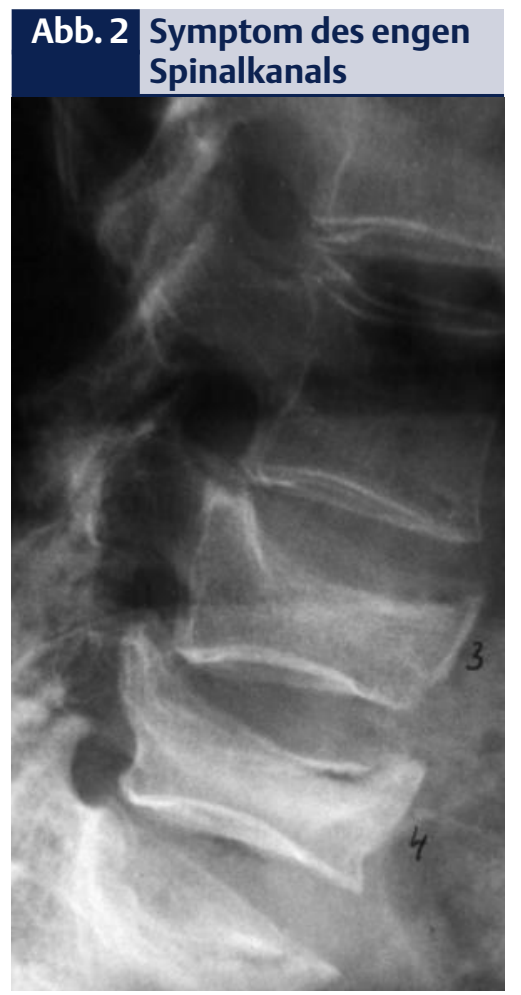

befähigt, zum anderen muss herausgestellt werden, dass es sich hierbei um nicht repräsentative Einzelfälle handelt. Durch gezielte Erhebungen mit standardisierten Befragungssystemen kann deutlich gemacht werden, dass Patienten mit fortgeschrittener Wirbelsäulenosteoporose oft behindert sind und hierdurch in eine soziale Isolation geraten.

\section{Hier nur einzelne Beispiele}

- Die Zeit, die zwischen der morgendlichen Erhebung und dem Niedersetzen zum Frühstück vergeht, umfasst bis zu $11 / 2$ Stunden. Bis zum Erreichen dieses Zieles werden mehrere Ruhepausen eingelegt, um der Entstehung zunehmender Schmerzen zu begegnen.

- Scheinbar banale Hausarbeiten können selbständig nicht mehr erledigt werden, wie zum Beispiel der Abwasch, das Aufräumen nach den Mahlzeiten, das Ziehen eines Staubsaugers durch die Wohnung, Bügeln oder Bettenmachen.

Lendenwirbelsäule zeigt breitflächige Deckplatteneinbrüche des 3. und 4. Lendenwirbelkörpers mit gleichzeitiger ventraler und dorsaler Höhenminderung der Wirbelkörper. Die dorsokraniale Kante von L3 und L4 ist nach hinten ausgelenkt und führt dadurch zu einer Einengung des Spinalkanals.

ten eine zugrunde liegende Osteoporose nicht festgestellt wird und eine spezifische Osteoporosetherapie unterbleibt, obwohl durch eine derartige Behandlung das Risiko zukünftiger Frakturen vorhersagbar und sehr eindrucksvoll gesenkt werden kann.

Mit zunehmender Zahl der Wirbelkörpereinbrüche erlebt die überwiegende Mehrzahl der Betroffenen einschneidende Einschränkungen der Leistungsfähigkeit im Rahmen der häuslichen Selbstversorgung (Abb. 2), jedoch auch im Rahmen beruflicher Tätigkeit. Immer wieder werden einzelne Patienten gesehen, die in eindrucksvoller Weise trotz multipler Wirbelkörpereinbrüche eine selbständige Lebensführung bewahren können. Zum einen ist völlig unklar, welche Mechanismen diese Menschen zu dieser Leistung wiederholten Ruhepausen während des Tages genötigt, um neu auftretenden Schmerzen zu entgehen. Wenn derartige Ruhepausen nicht eingelegt werden, entstehen Schmerzen und Beschwerden innerhalb von weniger als 5-10 Minuten und nehmen auch innerhalb weniger Minuten ein unerträgliches Ausmaß an.

- Reisen über längere Zeit mit Auto, Bahn oder gar Flugzeug sind nicht mehr möglich. Auch die Teilnahme an Veranstaltungen des täglichen Lebens (Kino, Theater, Konzert u.ä.) ist nicht möglich. Viele der fortgeschritten Erkrankten ziehen sich durch ihre Behinderung aus dem üblizurück.

- Mehr als 50\% der Patienten, die an einem Oberschenkelhalsbruch leiden, erleben trotz adäquater Therapie in Form von Operation und Rehabilitation keine Restitutio ad integrum (3). Der durchschnittliche lebensverkürzende Effekt eines Ober-
- Viele dieser Patienten sind zu chen gesellschaftlichen Leben 
Tab. 1 Risikofaktoren für eine glukokortikoid-induzierte Osteoporose

- Frakturen ohne nennenswertes Trauma

- Abnahme der Körpergröße um $>4 \mathrm{~cm}$ seit der Jugend oder $>2 \mathrm{~cm}$ seit der letzten Messung

- „Body-Mass-Index“ (BMI) $<20$ kg/m²

- Hohes Sturzrisiko

- Alter > 70 Jahre

- Stark eingeschränkte Mobilität

- Verkürzte Zeit der Hormonexposition bei Frauen (Alter bei Menopause minus Alter bei Menarche $<30$ Jahre)

schenkelhalsbruchs beträgt bei Patienten, die in der Mitte ihrer 70er Jahre betroffen werden, mehr als 7 Jahre und bei Patienten, die jenseits des 80. Lebensjahres einen derartigen Bruch erleiden mehr als 1 Jahr.

- Scharf steigt der Anteil derer an und erreicht deutlich mehr als $50 \%$, die nach Oberschenkelhalsbruch nicht mehr in der Lage sind, sich nach dem Aufstehen selbst zu versorgen (Bekleidung, Körperhygiene), frei über mehrere hundert Meter zu gehen oder Treppen zu steigen.

- Bei der Selbstversorgung wird mit zunehmender Krankheitsschwere ein zunehmender Anteil der Betroffenen von Fremdhilfe abhängig, weil regelmäßiger Einkauf nicht mehr möglich ist. Unbekannt ist die absolute Zahl der von Osteoporose betroffenen Patienten, die durch ihre Verluste der Leistungsfähigkeit in Alters- oder Pflegeheimen landen. Es wurde im Rahmen einer Fall-Kontroll-Studie gezeigt, dass 20\% der Patienten nach Oberschenkelhalsbruch im Laufe der zwölf dem Ereignis folgenden Monate versorgungspflichtig invalide werden (3).

\section{Folgekosten einer \\ Osteoporose}

Die prospektive Untersuchung einer Stichprobe in Deutschland ermittelte nach Oberschenkelhalsbruch für die Versorgung der Patienten (Akutbehandlung, Rehabilitation, gegebenenfalls Pflege) einen Finanzbedarf in der Größenordnung zwischen 15000 und 20000 Euro während der ersten zwölf dem Ereignis folgenden Monate (2). Diese Zahlen decken sich mit Zahlen aus Skandinavien, Großbritannien, den Beneluxländern und der Schweiz, wobei eine prospektive gesundheitsökonomische Studie in Genf zu dem Ergebnis kam, dass zumindest dort die Kosten deutlich oberhalb der hier angegebenen Zahlen lagen (7).

Es muss also für die Betreuung der Patienten mit Oberschenkelhalsbrüchen in Deutschland ein Betrag in der Größenordnung von 3 Milliarden Euro pro Jahr bereitgestellt werden. Felsenberg in Berlin hat hochgerechnet, dass die wirtschaftliche Gesamtbelastung im Rahmen aller Frakturen bei alt gewordenen Menschen im Bereich von 5 Milliarden Euro pro Jahr liegt.

\section{Pathophysiologie der \\ Knochenbrüche}

Das Frakturrisiko im Alter nimmt mit zunehmendem Lebensalter exponentiell zu. Frakturen treten zum einen auf, weil durch Osteoporose die mechanische Kompetenz der Knochen bei Belastungen beeinträchtigt ist. Frakturen treten auch auf, weil im Rahmen gehäuften Stürzens Knochen, dies gilt insbesondere bei hilflosem Sturz, mechanisch überlastet werden. In allen epidemiologischen Untersuchungen hat sich jedoch gezeigt, dass der für die Osteoporose charakteristische Verlust des Knochenmineralgehaltes ein von allen übrigen Risikofaktoren für Knochenbrüche unabhängiger Risikofaktor ist. Gleiches gilt für das Altern schlechthin, das ebenfalls bei allen bisher durchgeführten epidemiologischen Untersuchungen ein von allen anderen Faktoren unabhängiger Risikofaktor ist. Zu den Risikofaktoren der glukokortikoidinduzierten Osteoporose siehe auch Tabelle 1.

Dabei ist völlig unerheblich, ob der im Alter eintretende Verlust der Knochendichte als Alterserscheinung schlechthin interpretiert wird (wie dies gelegentlich auch heute noch geschieht), also als Alterserscheinung dem Erscheinen grauer Haare oder von Falten im Gesicht gleichgesetzt wird. Der entschei- dende Unterschied zwischen den üblichen und hinzunehmenden Alterserscheinungen und der mit dem Alter gehäuft auftretenden Osteoporose besteht darin, dass Haarausfall und Gesichtsfalten sozialmedizinisch und gesundheitsökonomisch bedeutungsarm sind, während die angebliche Alterserscheinung Knochensubstanzverlust von bedrängender sozialmedizinischer und gesundheitsökonomischer Bedeutung ist.

\section{Verschiedene Entitäten der Osteoporose}

Pathophysiologisch werden verschiedene Osteoporosen voneinander abgegrenzt. Mit zunehmender Kenntnis zur Biologie des Knochenstoffwechsels spaltet sich die scheinbar uniforme Krankheit in eine zunehmende Zahl verschiedener Entitäten auf.

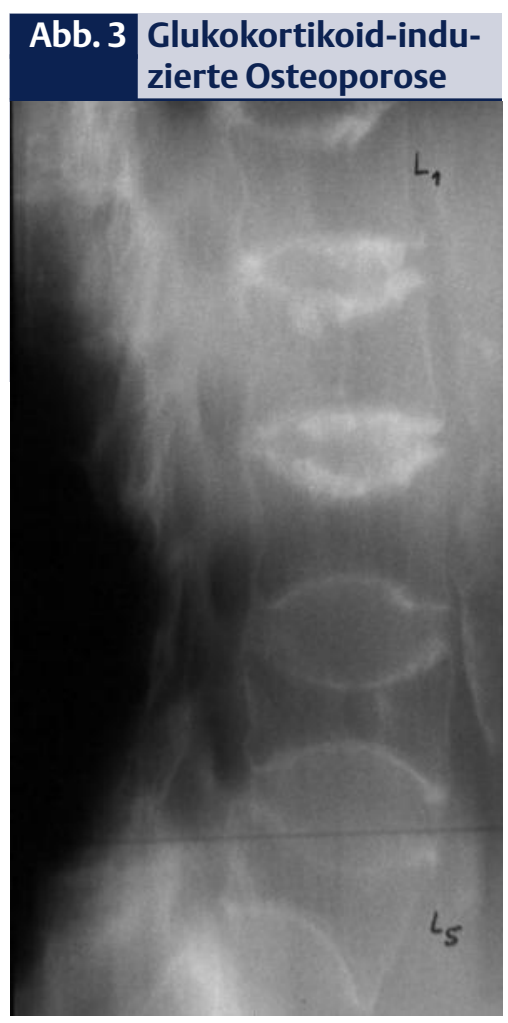

Die seitliche Röntgenaufnahme der LWS zeigt eine höchstgradig vermehrte Strahlentransparenz mit Ausbildung von Fischwirbeln. Die mattglasartige Auslöschung der Trabekelstruktur ist ein typischer Befund der glukokortikoid-induzierten Osteoporose. 


\section{Primäre Osteoporosen}

Im Alter entsteht, möglicherweise teilverursacht durch die im Alter zwangsläufig sich entwickelnde Sarkopenie (Verlust an Muskelmasse), eine Osteoporose. Einzelheiten zur Entstehung dieser Krankheitsform sind nach wie vor unbekannt, insbesondere ist ungeklärt, wieso nur ein Teil der alt werdenden Menschen hiervon betroffen ist. Es wird jedoch zunehmend deutlich, dass offensichtlich genetische Faktoren das Skelettschicksal im Alter vorherbestimmen. Hierfür spricht der Zusammenhang zwischen unterschiedlicher Knochendichte mit verschiedenen Ausprägungsformen (Allele) bei den Rezeptoren von Vitamin D, jedoch auch der Sexualhormone. Darüber hinausgehend scheint die Aktivität der verschiedenen Östrogenrezeptoren im Organismus von Bedeutung zu sein. So hat sich erst jüngst zeigen lassen, dass die durch mechanische Belastung beeinflussbare Ausformung der Knochen selbst von der Funktion von bestimmten Östrogenrezeptoren abhängt.

Ganz sicher existieren Osteoporoseformen, bei denen Erkrankungen und Funktionsstörungen der Osteoblasten zur Krankheitsursache werden. Ein klassisches Modell für diese Erkrankungsmöglichkeit stellen die verschiedenen Formen der Osteogenesis imperfecta dar, bei denen als Folge genetischer Störungen defekte Osteoblasten minderwertige und mechanisch instabile Kollagene herstellen, die den Knochenbruch begünstigen (8).

\section{Sekundäre Osteoporosen}

Dem die Wechseljahre der Frau verursachenden Mangel ovarieller Hormone wurde zurecht eine große Bedeutung bei der Entstehung der postmenopausalen Osteoporose zugewiesen, weil sich zeigen lässt, dass ein derartiger Hormonmangel den Knochenumsatz mit den Folgen gesteigerten Knochenabbaus anhebt. Wahrscheinlich ist auch beim Mann die Fähigkeit, aus Androgenen Östrogene zu bilden von Bedeutung für die Entstehung von Osteoporosen im Alter. Therapeutische Konse-

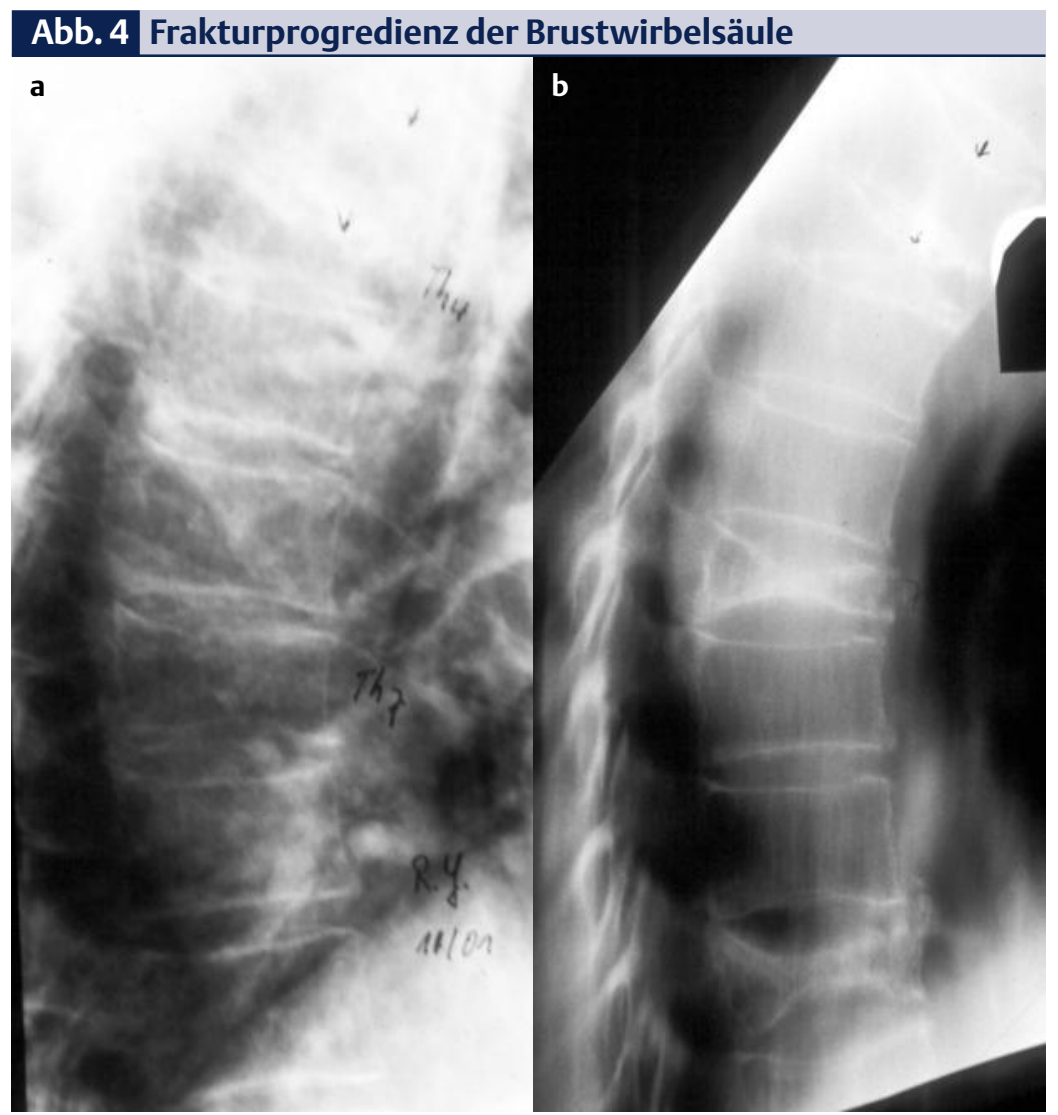

a) Die seitliche Röntgenaufnahme der BWS vom Oktober 2001 dokumentiert eine vermehrte Strahlentransparenz mit einem deutlichen Deckplatteneinbruch Th4 und einem Terminalplatteneinbruch mit zusätzlicher, geringer ventraler Höhenminderung bei Th7 im Sinne einer Fraktur.

b) Nur 3 Monate später ergibt die Verlaufskontrolle im Januar 2002 eine deutliche Frakturprogredienz mit nun einer Plattwirbelfraktur bei Th7 und einer neu hinzugekommenen Plattwirbelfraktur bei Th10.

quenzen werden hieraus zum jetzigen Zeitpunkt jedoch nicht mehr abgeleitet, weil sich zumindest beim Einsatz von Östrogen-/Gestagenpräparaten bei postmenopausalen Frauen zeigen ließ, dass diese zwar einerseits das Frakturrisiko senken, aber andererseits auch das Risiko für Herzinfarkt und Mammakarzinom erhöhen können. Wahrscheinlich hängt es auch hierbei von genetischen Faktoren $a b$, ob eine Frau durch Sexualhormonmangel in besonderem Maße durch Osteoporose bedroht wird. Wahrscheinlich gilt gleiches auch für den Mann, der in hohem Alter von relativem Sexualhormonmangel bedroht wird.

Von besonderer Bedeutung unter den sekundären Osteoporosen sind die im Rahmen einer Glukokortikoidtherapie entstehenden Erkrankungen. Dabei ist ebenfalls unklar, wieso bei einem Teil der steroidbehandelten Patienten auch nach jahrelanger Therapie mit zum Teil eindrucksvoll hohen Dosierungen eine Osteoporose nicht entsteht, während bei anderen innerhalb weniger Jahre nach Glukokortikoidbehandlung Osteoporosen zu Frakturen führen. Als mögliche Ursachen kommen sowohl die chronische, über mehrere Jahre andauernde Entzündung (Aktivierung der Osteoklasten durch Interleukine) als auch die Immobilisation im Rahmen der Grunderkrankung in Frage (Abb. 3, Abb. 4). Der Anteil derer, die unter länger dauernder Glukokortikoidtherapie eine Osteoporose mit Knochenbrüchen erleiden ist jedoch so hoch, dass in jedem Fall vor Beginn einer derartigen Behandlung eine osteologische Abklärung obligat ist. 


\section{Tab. 2 Knochendensitometrie}

- Empfohlene Messtechnik: Dual-Energy-X-rayAbsorptiometrie (sog. DEXA-Geräte der Hersteller Hologic, Lunar oder Norland)

- Indikation zur Messung vor Einleitung einer geplanten Glukokortikoidtherapie mit > 7,5 mg Prednisolonäquivalenten über voraussichtlich $>6$ Monate

- Verlaufskontrollen unter o.g. GlukokortikoidBehandlung alle 6-12 Monate

- Messort: Wirbelsäule bei Patienten < 75 Jahre und wenn möglich Hüfte; bei Patienten $>75$ Jahre sollte aufgrund von Messwertverfälschungen durch häufige degenerative Veränderungen an der LWS primär an der Hüfte gemessen werden

\section{Tab. 3 Basislabor bei der glukokortikoid- induzierten Osteoporose}

Im Vollblut:

- Blutkörperchensenkungsgeschwindigkeit (BSG)

- C-reaktives Protein (CRP)

Im Serum:

- Calcium

- Phosphat

- alkalische Phosphatase (aP)

- Gamma-glutamyl Transferase (GGT)

- Kreatinin (Crea)

- Immunfixationselektrophorese

- basales Thyreoidea-stimulierendes Hormon (TSH); Cave: TSH kann unter GlukokortikoidEinnahme vermindert sein

Auch die zur Steroidtherapie führenden chronisch entzündlichen Krankheiten können selbst zur Osteoporoseursache werden. Dies gilt sowohl für chronisch entzündliche Erkrankungen der Lunge und des Gastrointestinaltraktes, als auch für entzündliche Krankheiten des Bewegungsapparates.

Sekundäre Osteoporosen können auch Folge einer als Ausdruck endokrinologischer Krankheit angehobenen Glukokortikoidwirkung beim Morbus Cushing oder Cushingsyndrom sein. Der Anteil derer, die im Zusammenhang mit einer langzeitig bestehenden und unentdeckten und somit unbehandelten Hyperthyreose in der heutigen Zeit eine Osteoporose erleiden, dürfte begrenzt sein.

Unter den hämatologischen Systemkrankheiten, die eine Osteoporose erzeugen können, haben die systemischen Mastozytosen eine zunehmende Bedeutung gewonnen. Sie können Knochendestruktionen verursachen, ohne dass sie durch Hautsymptome (Urticaria pigmentosa) erkennbar wären. Auch ist das Ausmaß der weiteren möglichen Symptome (z.B. Flush-Syndrom, gastroenterologische Beschwerden oder anfallsartige Kopfschmerzen mit Übelkeit) variabel (8).

Selten, jedoch im Einzelfall von besonderer klinischer Bedeutung, sind schwangerschaftsassoziierte Osteoporosen, die bei einzelnen Patientinnen bereits zum Zeitpunkt der Entbindung oder kurze Zeit danach zu multiplen Wirbelkörpereinbrüchen führen.

Eine Reihe der als Risikofaktoren für Osteoporose angesprochenen Krankheiten erzeugen so genannte Mischosteopathien, bei denen der reine Knochenschwund mit einer Osteomalazie wechselnden Ausmaßes kombiniert ist (z.B. urämische Osteopathie, Osteopathie bei Pankreasinsuffizienz oder Sprue).

\section{- Diagnostik der Osteoporose}

Durch die Bestimmung des Knochenmineralgehaltes kann das Risiko zukünftiger Frakturen bei Osteoporose bestimmt werden. Zwar ist die Knochendichteanalyse hierdurch nur ein einzelner Mosaikstein bei der Abklärung einer Osteoporose, sie ist jedoch nach wie vor das einzige Verfahren, mit dessen Hilfe bei Patienten mit Osteoporose vor dem ersten Wirbelkörperbruch das Risiko zukünftiger Frakturen bestimmt werden kann. Das Ergebnis derartiger Analysen kann somit dazu beitragen, die Dringlichkeit einer spezifischen Therapie festzulegen.

Der Einsatz quantitativer Ultraschallverfahren ist nicht geeignet, die Indikation zum Beginn einer Therapie zu bestimmen. Der Grund ist nicht etwa, dass diese Verfahren im Hinblick auf ihre Vorhersagekraft von osteoporotischen Frakturen nicht ausreichend validiert wären, sondern dass die Übereinstimmung der Ergebnisse von Ultraschalluntersuchungen und von üblichen DEXAUntersuchungen außerordentlich begrenzt ist. Sämtliche Therapie- studien wurden jedoch bei Patienten durchgeführt, bei denen die Krankheit mittels DEXA-Methode diagnostiziert wurde. Somit bleibt streng genommen unklar, ob Patienten, bei denen die Diagnose mit Hilfe einer Ultraschalluntersuchung gestellt wurde, von einer derartigen Therapie in gleicher Weise profitieren können (8). Zum Einsatz der Knochendensitometrie siehe auch Tabelle 2.

Am schlechtesten validiert sind im Hinblick auf ihre Wertigkeit die quantitativen Computertomographieverfahren. Es ist zwar deutlich, dass eine feststellbare Reduktion des Knochenmineralgehaltes im Bereich des trabekulären Knochens unter 80 $\mathrm{mg} / \mathrm{cm}^{3}$ als Hinweis auf ein deutlich angehobenes Frakturrisiko zu interpretieren ist. Weitere Validierungen in Form prospektiver Studien erfolgten jedoch bisher nicht, und auch keine Validierung im Rahmen von Therapiestudien.

Laborchemische Analysen dienen dem Ausschluss sekundärer Osteoporosen (siehe oben) und werden nach üblichem Standard durchgeführt (Basislabor bei Osteoporose siehe Tab. 3). Bei bleibender Unklarheit sind auch histologische Untersuchungen adäquater Knochenbiopsien im Rahmen der Differentialdiagnostik einzusetzen.

Laboruntersuchungen (Bestimmungen des Knochenumsatzes) und histologische Untersuchungen sind dagegen unter Routinebedingungen nicht geeignet, die Auswahl von bei der Behandlung einzusetzenden Therapeutika zu erleichtern. Es schien in der Vergangenheit durchaus denkbar, dass der messbare Knochenumsatz Einfluss auf die Verordnung spezifischer Therapeutika nehmen konnte (z.B. osteoanabol wirksame Fluoride bei sog. „low-turnover" und antikatabol wirkende Bisphosphonate bei sog. „high-turnover“ Stoffwechselsituation der Knochen). Auch hat sich bei der Untersuchung von großen Patientengruppen durchaus zeigen lassen, dass eine prätherapeutisch festgestellte "high-turnover“-Situation ein Hinweis dafür sein kann, dass unter antikataboler BisphosphonatTherapie ein besonders deutlicher Knochendichtezuwachs erzielt wer- 
den kann. Bei der laborchemisch bestimmten Stoffwechselsituation ist jedoch die Streuung der Messwerte so groß, dass derartige Analysen beim einzelnen Patienten unter Routinebedingungen keine Aussagekraft haben (9). Eine Übersicht über den Einsatz der Marker zur Bestimmung des Knochenumsatzes gibt Tabelle 4 .

Auf der anderen Seite hat sich zeigen lassen, dass die heute zur Verfügung stehenden höchst wirksamen Medikamente ihre Wirkung relativ unabhängig vom anzunehmenden Knochenumsatz entfalten; darüber hinausgehend sind die Entscheidungsmöglichkeiten dadurch eingeschränkt, dass sich unter den bestklassifizierbaren Medikamenten (A-Klassifikation) ausschließlich antikatabol wirksame Medikamente befinden und damit ohnehin keine Wahlmöglichkeit besteht. Dies wird sich allerdings möglicherweise bereits in naher Zukunft durch die Einführung von Parathormon und von Strontiumranelat ändern, die beide als Osteoanabolika zu bewerten sind (10).

\section{Behandlungsoptionen}

Die Leitlinien des Dachverbandes deutschsprachiger osteologischer Fachgesellschaften (DVO) enthalten auch verbindliche Aussagen zur Therapie der glukokortikoidinduzierten Osteoporose sowie Aussagen zur Behandlung üblicher Osteoporoseformen, wie sie unabhängig von Glukokortikoiden allein als Folge chronisch entzündlicher Prozesse entstehen können (www. bergmannsheil/leitlinien-dvo).

Folgende Grundsätze sind dabei zu berücksichtigen:

1. Die Diagnose einer Osteoporose kann mit hinreichender Sicherheit unter Einsatz der heutzutage verfügbaren Diagnostika gestellt werden, auch ohne dass eine Fraktur als Spätkomplikation aufgetreten sein muss.

2. Die Therapie einer einmal festgestellten Osteoporose ist obligat, weil nur durch gezielte Therapie das zunehmende Risiko teiltraumatischer und atraumatischer Frakturen vorhersagbar gesenkt werden kann.
3. Es stehen Medikamente zur Verfügung, die in adäquater Weise im Rahmen prospektiver, randomisierter, kontrollierter Doppelblindstudien auf ihre Wirksamkeit überprüft wurden und deren Wirksamkeit durch diese Studien belegt wurde.

Die Existenz evidenzbasierter Leitlinien als Grundlage für Therapieentscheidungen hat zur Folge, dass sich verordnende Ärzte darauf berufen können, dass die von ihnen leitliniengerecht ausgewählte Behandlung medizinisch begründet ist, weil sie die Prinzipien einer auf Evidenz basierenden Medizin berücksichtigt (Verfügbarkeit adäquat überprüfter Medikamente; nachweisbarer Nutzen einer Therapie für die behandelten Patienten; gesundheitsökonomische Sinnhaftigkeit einer derartigen Behandlung). Dies wiederum schließt aus, dass derartige Verordnungen durch Budgetierungen verhindert werden. Schon jetzt ist in Deutschland erkennbar, dass Regressansprüche an Ärzte erfolgreich abgewehrt werden können, wenn diese wegen Verordnungen bei Patienten mit Osteoporose zustande kamen und wenn sich zeigen lässt, dass diese Verordnungen medizinisch begründet sind.

Neu ist inzwischen, dass nicht nur die bei der Osteoporose eingesetzten Pharmaka im Rahmen adäquater Studien auf ihre Wirksamkeit untersucht werden, sondern dass dieses auch für Hilfsmittel gilt, die bei Patienten mit Osteoporose eingesetzt werden können (z.B. Hüftprotektor „Safe-hip“ oder Rückenorthese „Spinomed“).

\section{Pharmakotherapie der Osteoporose}

Im Rahmen adäquater Studien wurde bei Normalpersonen gezeigt, dass Supplementation mit Calcium und Vitamin D das Risiko von Knochenbrüchen zu senken vermag. Diese Wirkung kommt nicht allein durch den Einfluss des Vitamin D auf den Knochenstoffwechsel zustande, sondern ist auch Folge der Vitamin D-bedingten Verbesserung der Muskelfunktion, die das Risiko frakturerzeugender Stürze senkt (11). Wenn

\section{Tab. 4 Marker des Knochenumsatzes}

- Aufgrund hoher Messvarianzen keine Indikation für die klinische Routine

- Mögliche Hinweisgebung zur differentialdiagnos tischen Abklärung von Sekundärosteoporosen

- Verminderung der so genannten Abbaumarker unter Therapie mit Bisphosphonaten oder Raloxifen. Als Abbaumarker werden Eiweiße bezeichnet, die beim Knochenabbau aus dem Kollagenstoffwechsel gebildet werden und renal ausgeschieden werden (z.B. Desoxy-Pyridinoline oder N-terminales Telopeptid des Typ 1 Kollagens)

also zusätzlich zur festgestellten Osteoporose keine Störung der Calciumhomöostase vorliegt, die eine Hyperkalzämie verursacht, ist die Therapie mit Calcium und Vitamin D als Behandlungsbasis einzustufen.

Für die Therapie postmenopausaler und seniler Osteoporosen verdienen die Bisphosphonate Fosamax ${ }^{\circledR}$ (10 mg pro Tag) und Actonel ${ }^{\circledR}(5 \mathrm{mg}$ pro Tag) beste Bewertung (A-Klassifikation). Aufgrund von Äquivalenzüberlegungen und begründet durch Studien kann davon ausgegangen werden, dass beide Präparate auch in abgewandelter Applikationsform (Fosamax ${ }^{\circledR}$ : $70 \mathrm{mg}$ pro Woche und Actonel ${ }^{\circledR}$ : $35 \mathrm{mg}$ pro Woche) gleich wirksam sind.

Der selektive Östrogen-RezeptorModulator ("Selective-Estrogen-Receptor-Modulator“ oder „SERM“) Raloxifen (Evista ${ }^{\circledR}$ ) wird in seiner Dosis von $60 \mathrm{mg} /$ Tag identisch bewertet, ist aber für Männer nicht geeignet (12).

Während die Supplementation mit Calcium und Vitamin D lebenslang erfolgen sollte, empfehlen wir, die hier genannten spezifischen Medikamente bei Patienten, die noch keine Fraktur im Rahmen ihrer Osteoporose erlitten haben, über einen Zeitraum von drei Jahren einzusetzen. Bei Patienten jedoch, die bereits Frakturen als Spätkomplikation ihrer Osteoporose erlitten haben, sollte eine Behandlung über fünf Jahre durchgeführt werden, da sich insbesondere für das Bisphosphonat Alendronat (Fosamax ${ }^{\circledR}$ ) zeigen ließ, dass auch nach mehr als drei Jahren Behandlung ein Zuwachs an Knochendichte einhergehend mit einem verminderten Knochenbruchrisiko erreicht wird. 


\section{Abb. 5 Prophylaxe der glukokortikoid- induzierten Osteoporose}

(ab 7,5 mg Prednisolonäquivalent pro Tag

über mehr als 6 Monate)

Knochendichte (KD) LWS oder Hüfte

T-Score größer -1,0 SD:

- Calcium 1000 mg/Tag

- Vitamin D 1000 I.E./Tag

- Kontrolle KD-Messung in $1 \mathrm{Jahr}$
Für den Mann mit Osteoporose ist Fosamax ${ }^{\circledR}$ in einer Dosis von 10 mg pro Tag offiziell als Therapeutikum zugelassen. Auch hier erlauben Äquivalenzüberlegungen jedoch die Schlussfolgerung, dass Fosamax ${ }^{\circledR}$ auch bei einmaliger Gabe von $70 \mathrm{mg}$ pro Woche wirksam ist.

Zur Behandlung speziell der glukokortikoid-induzierten Osteoporose bei postmenopausalen Frauen ist in Deutschland das Bisphosphonat Risedronat (Actonel ${ }^{\circledR}$ ) offiziell zugelassen. Aufgrund der vorliegenden Datenlage aus klinischen Studien kann allerdings davon ausgegangen werden, dass auch das Bisphosphonat Alendronat (Fosa$\left.\max ^{\circledR}\right)$ in gleicher Weise wirksam ist, wenngleich - formal betrachtet - die Gabe von Fosamax ${ }^{\circledR}$ bei Patienten mit glukokortikoid-induzierter Osteoporose eine so genannte „offlabel“-Indikation darstellt (12).

Ein mögliches Vorgehen, sowohl hinsichtlich der Prophylaxe als auch der Therapie der glukokortikoidinduzierten Osteoporose, ist in den Abbildungen 5 und 6 dargestellt.

Beim Vorliegen von Kontraindikationen gegen den Einsatz oraler Bisphosphonate (z.B. Divertikel der Speiseröhre oder Ösophagusvarizen bei gleichzeitig bestehender Leberzirrhose) können ersatzweise intravenöse Bisphosphonate eingesetzt werden. Für das Bisphosphonat Ibandronat (Bondronat ${ }^{\circledR}$ ) ist ein adäquater Wirkungsnachweis bei oraler Gabe erbracht worden, wobei Äquivalenzüberlegungen die Annahme rechtfertigen, dass das Präparat auch in seiner intravenösen Applikationsform wirksam ist. Gleichwohl lehnen Krankenkassen aufgrund ihrer Beratung durch den Medizinischen Dienst gelegentlich die Kostenüber- nahme für derartige Präparate ab. In solchen Fällen ist der durch eine Fehlbewertung der Situation herbeigeführten Kassenentscheidung zu widersprechen und im Zweifelsfall der Rechtsweg einzuschlagen, da durch ein kürzlich ergangenes Urteil des Bundessozialgerichtes die Durchführung einer wirksamen Behandlung bei bedrohlichen Krankheiten auch außerhalb der formalen Zulassung zu Lasten der gesetzlichen Krankenkassen möglich ist.

Nur noch den Charakter von Reservepräparaten haben fluoridhaltige Medikamente sowie Calcitonine, weil ihre Wirkung im Rahmen adäquater Studien bisher nicht überprüft wurde beziehungsweise entsprechende Studien zu widersprüchlichen Ergebnissen geführt hatten (12).

Durch eine sexualhormonersetzende Therapie kann bei der Frau das Risiko zukünftiger Frakturen gesenkt werden. Der Einsatz einer kombinierten Östrogen-GestagenSubstitutionstherapie gilt jedoch inzwischen aufgrund der Ergebnisse der WHI ${ }^{1}$-Studie als obsolet, da die beobachteten Nebenwirkungen (Anhebung des Risikos kardiovaskulärer Komplikationen wie Lungenembolien und Schlaganfälle, sowie ein Anstieg des Risikos für das Mammakarzinom) einen Einsatz nicht mehr rechtfertigen (12).

\section{Nichtpharmakologische \\ Maßnahmen}

Bei Patienten mit Osteoporose bei denen eine Anhebung des Sturzrisikos dokumentierbar ist (mehr als zwei Stürze während der vergangenen zwölf Monate), entspricht die Verordnung eines „Aufprallschutzes“ (Hüftprotektor „Safe-hip“) den
Prinzipien einer „Evidenz-basedmedicine“, weil im Rahmen adäquater Studien gezeigt wurde, dass die Nutzung das Risiko von Oberschenkelhalsbrüchen relevant zu senken vermag. Problematisch ist nach wie vor die begrenzte Compliance der Patienten (13).

Ursprünglich wurden Orthesen bei Patienten mit Osteoporose nur zögerlich verordnet, weil befürchtet werden musste, dass ihr Einsatz $\mathrm{zu}$ relativer Immobilisierung, $\mathrm{Ab}$ schwächung der Muskelkraft und Verstärkung der Osteoporose führen würde. Im Rahmen einer prospektiven, randomisierten, „cross-over“Studie zeigte sich jedoch, dass die

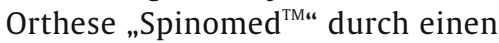
zugrunde liegenden BiofeedbackMechanismus entgegengesetzt der Erwartung zu einem dauerhaften Training der Rumpfmuskulatur führt (14). Die Muskelkraft nimmt sowohl im Bereich der Extensoren als auch der Flexoren relevant zu. Dies senkt messbar die Schwankneigung des Körpers, wobei in diesem Zusammenhang anzumerken ist, dass hohe Schwankneigung ein validierter Risikofaktor für zukünftige Stürze und Frakturen ist. Darüber hinausgehend werden durch Nutzung der Orthese sowohl messbare Schmerzen als auch abfragbare Aktivitäten des täglichen Lebens verbessert. Diese Orthese muss „einschleichend" eingesetzt werden und darf anfangs nur stundenweise getragen werden, weil das initiierte Muskeltraining sonst $\mathrm{zu}$ Überlastungserscheinungen führt (Abb. 7) (14).

Rehabilitationsbehandlung

Die Paragraphen 1-4 des IX. Sozialgesetzbuches beschreiben ein-

\section{Abb. 6 Therapie der glukokortikoid-induzierten Osteoporose}

(ab 7,5 mg Prednisolonäquivalent pro Tag über mehr als 6 Monate)

Knochendichte (KD) LWS oder Hüfte

\begin{tabular}{ll}
\hline T-Score zwischen -1,0 und -2,5 SD: & T-Score kleiner -2,5 SD: \\
- Calcium $1000 \mathrm{mg} /$ Tag & - Calcium $1000 \mathrm{mg} /$ Tag \\
- Vitamin D 1000 I.E./Tag & - Vitamin D 1000 I.E./Tag \\
oder & - Alendronat $10 \mathrm{mg} / \mathrm{Tag}$ \\
- Alfacalcidol $1 \mu \mathrm{g} / \mathrm{Tag}$ & oder \\
und & - Risedronat $5 \mathrm{mg} /$ Tag \\
- Kontrolle KD-Messung in $1 / 2 \mathrm{Jahr}$ & - Kontrolle KD-Messung in $1 \mathrm{Jahr}$
\end{tabular}


deutig, dass ein gesetzlicher Anspruch auf Rehabilitationsmaßnahmen besteht, wenn Personen krankheitsbedingt bedroht sind, Behinderungen über einen Zeitraum von mehr als sechs Monaten zu erleiden. Die Rehabilitation dient dabei nicht der Erzeugung optimaler Verhältnisse (soll also nicht instand setzen, in Zukunft das Matterhorn zu besteigen), sondern dazu, den Patienten in das übliche soziale Leben zu integrieren.

Die oben dargestellte Symptomatologie bei fortschreitender Osteoporose begründet dabei einen Rehabilitationsanspruch zweifelsfrei. Auch hier kommt es immer wieder zur Ablehnung von Kostenübernahmeerklärungen durch die Kostenträger aufgrund der Beratung durch Medizinische Dienste. Auch in derartigen Fällen ist bei solchen fehlgeleiteten Entscheidungen Widerspruch einzulegen, im Zweifel ist der Rechtsweg einzuschlagen.

\section{Rehabilitationsziele (13)}

1. Erlernen des adäquaten Umganges mit einer chronisch behindernden Krankheit durch entsprechende Gesundheitserziehung und psychologische Betreuung.

2. Einfluss auf Beschwerdeausmaß und Schmerzen durch Einsatz physikalisch balneologischer und krankengymnastischer Maßnahmen.

3. Mobilisierung mit dem Ziel, Handlungsfähigkeiten zu verbessern und den Aktionsradius auszudehnen.

4. Ergotherapie mit dem Ziel, die Möglichkeiten einer von Fremdhilfe unabhängigen Selbstversorgung zu verbessern.

Bei Einweisung in eine Rehabilitationsklinik ist darauf zu achten, dass die gewählte Einrichtung in besonderem Maße für die Behandlung von Patienten mit Osteoporosen spezialisiert ist.

\section{Summary}

Currently in Germany some two million women and more than 800,000 men have vertebral fractures. $40 \%$ of the female patients with rheumatoid

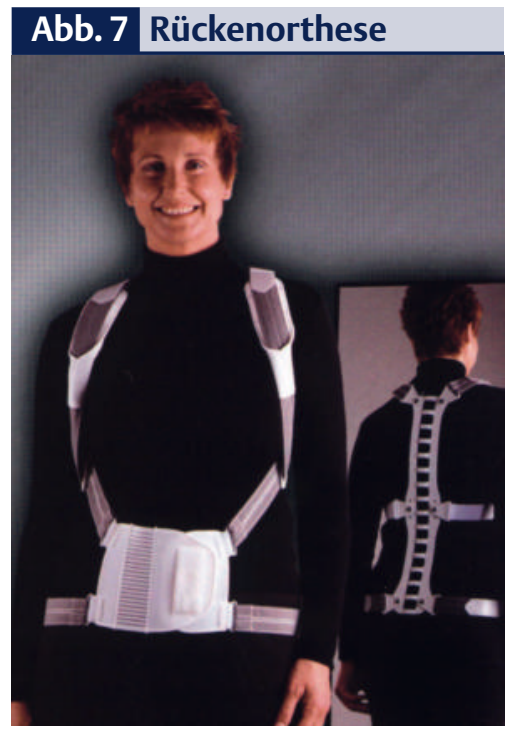

Die auf dem so genannten „Rucksackprinzip“ aufbauende Rückenorthese „Spinomed ${ }^{\mathrm{TM}}$ “ dient der Aufrichtung der Wirbelsäule vor allem nach Wirbelkörpersinterungen bei Osteoporose. Sie besteht aus einer kalt verformbaren Rückenpelotte, die durch ein Gurtsystem mit Klettverschlüssen der Form der Wirbelsäule individuell angepasst werden kann.

arthritis (RA) also have osteopenia, $20 \%$ osteoporosis. $80 \%$ of all patients with $R A$ are older than 50 years. In an unknown percentage of the victims, vertebral fractures are associated with the worst imaginable pain and result in massive impairment of domestic self-care.

Bone density analysis is only a single element of an investigation of osteoporosis, but remains the sole procedure with the aid of which the risk of future fractures can be estimated. By way of treatment options, medicaments and non-pharmacological measures listed in the guidelines of the umbrella organization of German-speaking osteological societies (DVO) are available.

Literatur

1. Felsenberg D, Silman AJ, Lunt M et al. for the European Prospective Osteoporosis Study (EPOS) Group. Incidence of vertebral fracture in Europe: results from the European Prospective Osteoporosis Study (EPOS). J Bone Miner Res 2002; 17: 716-724

2. Pfeifer M, Wittenberg R, Würtz R, Minne HW. Schenkelhalsfrakturen in Deutsch- land. Dt. Ärzteblatt 2001; 98: A17511757

3. Schürch MA, Rizzoli R, Mermillod B, Vasay H, Michel JP, Bonjour JP. A prospective study on socio-economic aspects of fracture of the proximal femur. J Bone Miner Res 1996; 11: 1935-1942

4. Schlaich C, Minne HW, Bruckner T et al. Reduced pulmonary function in patients with spinal osteoporotic fractures. Osteoporos Int 1998; 8: 261-267

5. Kado DM et al. Mortality after vertebral fractures. Arch Intern Med 1999; 159: 1215-1220

6. Leidig-Bruckner G, Minne HW, Schlaich C et al. Clinical grading of spinal osteoporosis: quality of life components and spinal deformity in women with vertebral osteoporosis. J Bone Miner Res 1997; 12: 663-675

7. Lippuner K, van Overbeck J, Perrelet R, Bosshard $\mathrm{H}$, Jaeger $\mathrm{PH}$. Incidence and direct medical costs of hospitalizations due to osteoporotic fractures in Switzerland. Osteoporos Int 1997; 7: 414-425

8. Pollähne W, Grieser T, Pfeifer M, Minne HW. Diagnostik und Differentialdiagnostik primärer und sekundärer Osteoporosen. Stuttgart New York: Georg Thieme Verlag, 1996

9. Pollähne W, Burckhardt P, Bröll H, Delling G, Minne HW. Therapie und Differentialtherapie primärer und sekundärer Osteoporosen. Stuttgart New York: Georg Thieme Verlag, 1999

10. Pfeifer M, Lehmann R, Minne HW. Die Therapie der Osteoporose aus dem Blickwinkel einer auf Evidenz basierenden Medizin. Med Klin 2001; 96: 270-80

11. Pfeifer M, Begerow B, Minne HW, Abrams C, Nachtigall D, Hansen C. Effects of a short-term vitamin $\mathrm{D}$ and calcium supplementation on body sway and secondary hyperparathyroidism in elderly women. J Bone Miner Res 2000; 15: 1113-1118

12. Pfeifer M, Minne HW. Evidenzbasierte Therapie der Osteoporose. Dtsch Med Wochenschr 2003;128:931-4.

13. Pfeifer M, Begerow B, Minne HW. Rehabilitation bei Osteoporose. Dtsch Med Wochenschr 2003; 128: 941-945

14. Pfeifer M, Begerow B, Minne HW. Randomized trial of effects of a newly developed spinal orthosis on posture, trunk muscle strength, and quality of life in women with postmenopausal osteoporosis. Am J Phys Med Rehabil 2004; 86: (in print)

\section{Anschrift für die Verfasser}

Dr. med. Michael Pfeifer

Institut für Klinische Osteologie „Gustav

Pommer“ und Klinik „Der Fürstenhof“

Am Hylligen Born 7

31812 Bad Pyrmont

Fax: $05281 / 151100$

eMail: iko_pyrmont@t-online.de 